\title{
Experimental test of quantum no-hiding theorem
}

\author{
Jharana Rani Samal * \\ Dept. of Physics and NMR Research Centre, \\ Indian Institute of Science,Bangalore, India \\ Arun K. Pati \\ Institute of Physics, Sainik School Post, \\ Bhubaneswar-751005, Orissa, India \\ Anil Kumar \\ Dept. of Physics and NMR Research Centre, \\ Indian Institute of Science,Bangalore, India
}

(Dated: February 6, 2018)

\begin{abstract}
Linearity and unitarity are two fundamental tenets of quantum theory. Any consequence that follows from these must be respected in the quantum world. The no-cloning theorem [1] and the no-deleting theorem [2] are the consequences of the linearity and the unitarity. Together with the stronger no-cloning theorem they provide permanence to quantum information [3], thus, suggesting that in the quantum world information can neither be created nor be destroyed. In this sense quantum information is robust, but at the same time it is also fragile because any interaction with the environment may lead to loss of information. Recently, another fundamental theorem was proved, namely, the no-hiding theorem [4] that addresses precisely the issue of information loss. It says that if any physical process leads to bleaching of quantum information from the original system, then it must reside in the rest of the universe with no information being hidden in the correlation between these two subsystems. This has applications in quantum teleportation [5], state randomization [6], private quantum channels [7], thermalization [8] and black hole evaporation [9]. Here, we report experimental test of the no-hiding theorem with the technique of nuclear magnetic resonance (NMR). We use the quantum state randomization of a qubit as one example of the bleaching process and show that the missing information can be fully recovered up to local unitary transformations in the ancilla qubits. Since NMR offers a way to test fundamental predictions of quantum theory using coherent control of quantum mechanical nuclear spin states, our experiment is a step forward in this direction.
\end{abstract}

\footnotetext{
* Deceased on her 27th birthday 12th Nov. 2009. The experimental work of this paper was completely carried out by the first author. We dedicate this paper to the memory of the brilliant soul of Ms. Jharana Rani Samal.
} 
There are many physical processes in nature which lead to apparent loss of information. The examples can be cited starting from quantum state randomization to thermalization, black hole evaporation and so on. For example, in the thermalization process a physical system starts from an arbitrary state and after the interaction with a heat bath it thermalizes to a canonical thermal distribution. Similarly, in the black hole evaporation a pure quantum state transforms to a thermal mixed state that has no information about the original pure state [9]. Another situation where information about the original system may be lost is the decoherence [10]. The process of decoherence is ubiquitous in the quantum world. When a quantum system in a pure state interacts with the environment they get entangled and in this process the original system looses its purity and becomes a mixed state. Typically, the system looses its phase coherence, but it might happen that after interaction with the environment the system ends up being in a mixed state that has no information about the original. If the original information about the system has disappeared then one may wonder where it has gone? This question has bothered many scientist. In the classical world, if information is missing from some system then it may happen in two ways. Classical information can be completely hidden from a subsystem if it is moved to another location, or it may be encoded as correlations between a pair of subsystems like in the Vernam cipher (one-time pad) [11]. In general, any information at classical level will be hidden as a combination of these two [12]. But we know that at a fundamental level quantum theory is at work. Using the laws of quantum theory it can be shown that if the information is missing from one system then it simply goes and remains in the rest of the subsystem which may be regarded as the environment or the universe. The missing information cannot be hidden in the correlations between the system and the environment. Unlike classical information, quantum theory allows only one way to completely hide quantum information, that is by moving it to the remaining subsystems [4].

Any physical process that bleaches out the original information we call it a 'hiding' process. Consider a physical process which transforms an arbitrary pure state $\rho=|\psi\rangle\langle\psi|$ to a fixed mixed state $\sigma$ that has no dependence on the input state. Thus, the hiding process maps $\rho \rightarrow \sigma$ with $\sigma$ fixed for all $\rho$. Let $\sigma$ has a spectral decomposition as $\sigma=\sum_{k} p_{k}|k\rangle\langle k|$, where the $p_{k}$ are the nonzero eigenvalues with $\sum_{k} p_{k}=1$ and the orthonormal set $|k\rangle$ are the eigenvectors. Note that this process is a generalization of the Landauer erasure map. In the erasure process any pure state is transformed to a fixed pure state $|0\rangle$, i.e., $|\psi\rangle\langle\psi|\rightarrow| 0\rangle\langle 0|$ (the resetting operation) [13]. In the hiding process if the final state is such that we have all $p_{k}$ 's are zero except one then we have the erasure process. Therefore, this generalizes the Landauer erasure to any hiding process (not merely erasure).

We know that any physical process can be thought of as a unitary process in an enlarged Hilbert space by attaching an ancilla. Therefore, the hiding process can be represented as a unitary transformation from a pure state $|\psi\rangle$ to another pure state $|\Psi\rangle$ such that $\operatorname{Tr}_{\mathrm{A}}(|\Psi\rangle\langle\Psi|)=\sigma$. The hiding process that bleaches out the original information can be expressed in terms of the Schmidt decomposition of the final state as

$$
|\psi\rangle \rightarrow|\Psi\rangle=\sum_{k=1}^{K} \sqrt{p_{k}}|k\rangle \otimes\left|A_{k}(\psi)\right\rangle,
$$

where $p_{k}$ are the $K$ non-zero eigenvalues of the density matrix $\sigma,\{|k\rangle\}$ are its eigenvectors and $\left\{\left|A_{k}\right\rangle\right\}$ are the orthonormal states of the ancilla. Using the linearity and the unitarity 
of quantum mechanics one can show that the final state must be of the following form [4]

$$
|\Psi\rangle=\sum_{k} \sqrt{p_{k}}|k\rangle \otimes\left(\left|q_{k}\right\rangle \otimes|\psi\rangle \oplus 0\right)
$$

where $\left\{\left|q_{k}\right\rangle\right\}$ is an orthonormal set of $K$ states and $\oplus 0$ denotes the fact that we substitute any unused dimensions of the ancilla space by zero vectors. This tells us that under this hiding map the pure state $|\psi\rangle$ moves to the ancilla. Since we are free to swap $|\psi\rangle$ with any other state in the ancilla using purely local unitary operations in the ancilla Hilbert space, one can say that any information about $|\psi\rangle$ that is encoded globally is in fact encoded entirely within the ancilla. Moreover, the quantum information contained in $|\psi\rangle$ cannot be encoded in the bipartite correlations of the system and the ancilla. This is the no-hiding theorem in quantum theory [4].

The simplest example of a hiding process is the quantum state randomization where an arbitrary pure state in a $d$-dimensional Hilbert space transforms to a completely mixed state, i.e., $|\psi\rangle\langle\psi| \rightarrow \frac{I}{d}$, where the final density matrix is same for all input states. The exact randomization of an arbitrary pure state of dimension $d$ can be performed with an ancilla of dimension at least $d^{2}$. For a single qubit, the randomization operation can be performed in a unitary manner by attaching an ancilla of Hilbert space dimension four (by using two qubits). State randomization has cryptographic applications, like, in private transmission of quantum information using a shared classical key [7]. For example, if Alice encrypts a message by applying some invertible quantum operation to some state and converts it to a fixed state, then Eve has no chance of knowing the original state. Bob who has access to the key can recover the message state by applying the invertible quantum operation.

Consider an arbitrary single qubit state $|\psi\rangle=\alpha|0\rangle+\beta|1\rangle$, where $\alpha$ can be chosen to be real and $\beta$ a complex number. According to the state randomization for any input state of a qubit the output state will be a random mixture. In this case the physical map is a completely positive map as given by

$$
|\psi\rangle\left\langle\psi\left|\rightarrow \frac{1}{4} \sum_{k=0}^{3} \sigma_{k}\right| \psi\right\rangle\langle\psi| \sigma_{k}=\frac{I}{2},
$$

where $\sigma_{0}=I$ and $\sigma_{k},(k=1,2,3)$ are the Pauli matrices. The above map can be thought of as a unitary map by attaching two qubits as the ancilla. The unitary transformation is given by

$$
|\psi\rangle|A\rangle \rightarrow|\Psi\rangle=\frac{1}{2} \sum_{k=0}^{3} \sigma_{k}|\psi\rangle\left|A_{k}\right\rangle,
$$

where $\left|A_{k}\right\rangle$ are orthonormal and the initial state of the ancilla is an equal superposition of all computational basis states, i.e.,

$$
|A\rangle=\frac{1}{2} \sum_{k=0}^{3}\left|A_{k}\right\rangle=\frac{1}{2}(|00\rangle+|01\rangle+|10\rangle+|11\rangle) .
$$

The unitary operator that realizes the above randomization operation is a conditional unitary operation (a three qubit gate) which is given by

$$
U=\sum_{k=0}^{3} \sigma_{k} \otimes\left|A_{k}\right\rangle\left\langle A_{k}\right|
$$




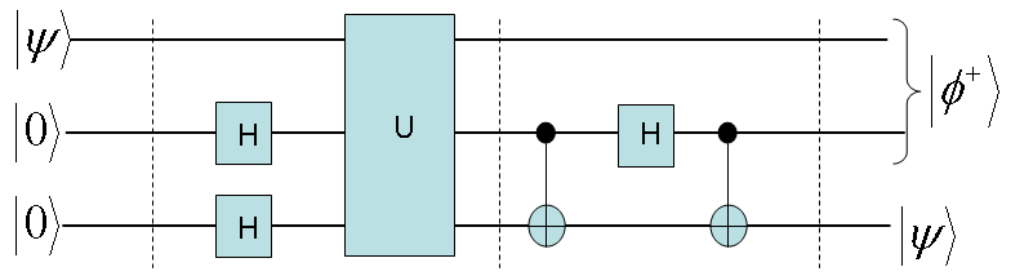

FIG. 1: Circuit diagram for testing the no-hiding theorem using the state randomization. U is the unitary operator for randomization. $\mathrm{H}$ represents the Hadamard gate. Dots and circles represent the CNOT gates. After randomization, the state $|\psi\rangle$ on the first qubit has been transferred to the second ancilla qubit (up to local unitary transformation in the ancilla).

We can see that if we trace out the ancilla qubits from the final state given in (4) we do get a completely mixed state. Now, the important question is where has the missing information gone that has bleached out from the original qubit. The no-hiding theorem provides answer to this question.

In this simplest example of quantum bleaching process, we will see that the missing information is simply residing in the two qubit ancilla state (up to local unitary transformation in the ancilla Hilbert space). This is the essence of the no-hiding theorem: the hidden information is fully encoded in the remainder of the Hilbert space. To reconstruct the original quantum information $|\psi\rangle$ from the ancilla qubits we need two CNOT gates and one Hadamard gate [Fig. 1]. Now, if we apply the following ancilla local unitary $U_{23}=\mathrm{CNOT}_{23}\left(I_{2} \otimes H_{3}\right) \mathrm{CNOT}_{23}$ on the pure state $|\Psi\rangle$ we get

$$
\begin{aligned}
|\Psi\rangle & =\frac{1}{2} \sum_{k=0}^{3} \sigma_{k}|\psi\rangle\left|A_{k}\right\rangle \rightarrow \frac{1}{2} \sum_{k=0}^{3} \sigma_{k}|\psi\rangle U_{23}\left|A_{k}\right\rangle=\mathrm{CNOT}_{23}\left(I_{2} \otimes H_{3}\right) \mathrm{CNOT}_{23}|\Psi\rangle \\
& =\frac{1}{\sqrt{2}}(|00\rangle+|11\rangle)|\psi\rangle=\left|\Psi_{\text {out }}\right\rangle .
\end{aligned}
$$

From (7) we can see that the first and the second qubits are in the Bell state and the third qubit contains the original information. Thus, the missing information can be fully recovered from the ancilla in intact form with no information being hidden in the correlation between the system and the ancilla. This shows that the no-hiding theorem is indeed respected in the state randomization process.

In the sequel we give details of the experimental implementation of the quantum state randomization. Liquid state NMR has been successfully used as a test bed for a large number of quantum information protocols including Grover's algorithm [14], Shor's algorithm [15], quantum teleportation [16], adiabatic quantum computation [17, 18], estimation of the ground state of Hydrogen atom up to 45 bits [19] and more recently experimental verification of the non-destructive discrimination of Bell-states [20]. Here, we report an experimental verification of the quantum no-hiding theorem using NMR. Experiments have been performed in a three qubit hetero-nuclear spin system formed by the ${ }^{1} \mathrm{H},{ }^{19} \mathrm{~F}$ and ${ }^{13} \mathrm{C}$ nuclei of ${ }^{13} \mathrm{C}$-enriched dibromo fluoro methane $\left({ }^{13} \mathrm{CHFBr}_{2}\right)$ [21]. Fig. 2(a) shows the equilibrium spectrum for the three nuclei at $300 \mathrm{~K}$ recorded in a Bruker AV500 spectrometer, where the resonance frequencies of ${ }^{1} \mathrm{H},{ }^{19} \mathrm{~F}$ and ${ }^{13} \mathrm{C}$ are $500 \mathrm{MHz}, 470 \mathrm{MHz}$ and $125 \mathrm{MHz}$ respectively. We have taken ${ }^{1} \mathrm{H},{ }^{19} \mathrm{~F}$ and ${ }^{13} \mathrm{C}$ as the first, the second and the third qubit, respectively. 


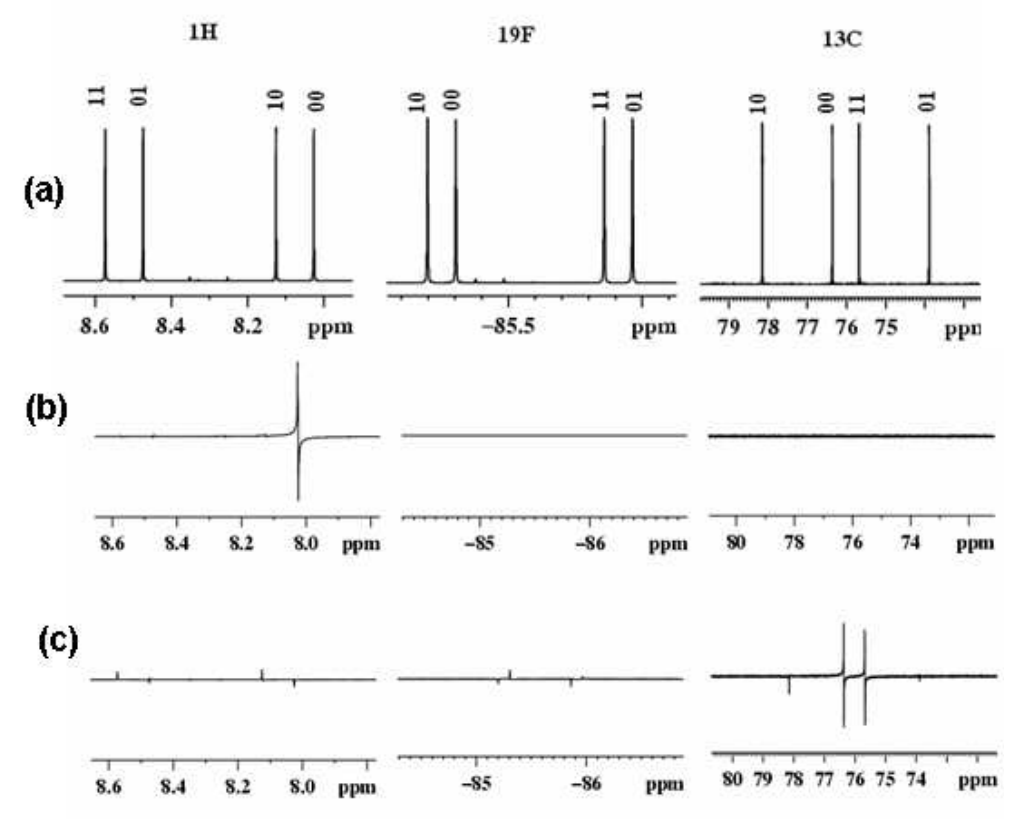

(d)

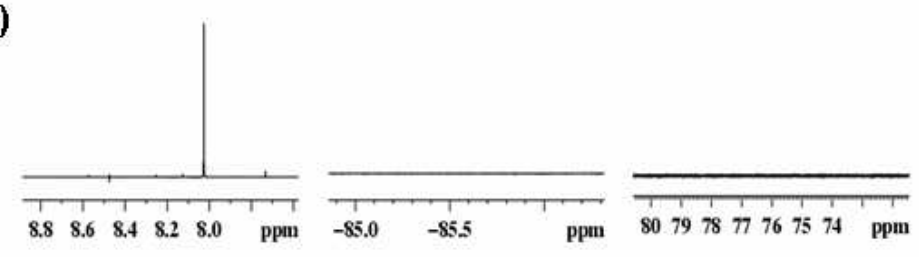

(e)

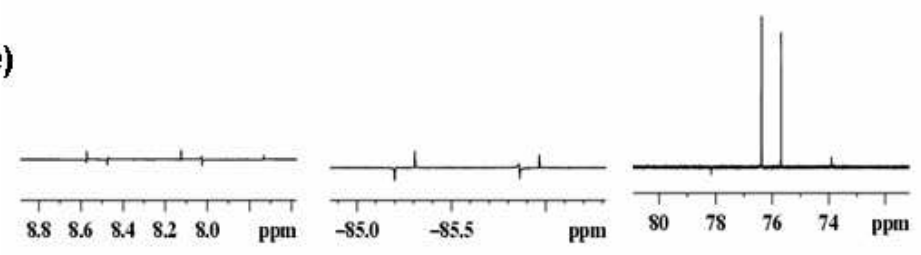

FIG. 2: (a)The equilibrium spectra of ${ }^{13} \mathrm{CHFBr} 2$ dissolved in acetone-d6 at $300 \mathrm{~K}$ on a AV500 NMR spectrometer. Labelling of the transition is given on top of each spectral line. The scalar coupling between different spins are measured as $J_{H C}=224.5, J_{F C}=-310.9, J_{H F}=49.7$. For $\theta=\pi / 2$ and $\phi=0,(\mathrm{~b})$ and (c) are respectively the experimental spectra for the input $\left(|\psi\rangle_{1}|00\rangle_{23}\right)$ and output $\left(\left|\phi^{+}\right\rangle_{12}|\psi\rangle_{3}\right)$ state for the 3 spins. The receiver phase is set using a separate experiment so that y magnetization appears as positive absorption mode. For $\theta=\phi=\pi / 2$, (d) and (e) are the experimental spectra showing respectively the input and output states for the 3 spins .

Fig. 1 depicts the circuit diagram that implements the state randomization for a single qubit in a unitary manner. Using the quantum circuit of Fig. 1, an equivalent NMR pulse sequence has been developed here (Fig. 3). This pulse sequence contains several elements namely: (i) the preparation of the pseudo pure state (PPS) [21], (ii) the process of initialization, (iii) the randomization operation using its unitary extension, (iv) extraction of the original quantum information from the ancilla by applying local unitary transformations 


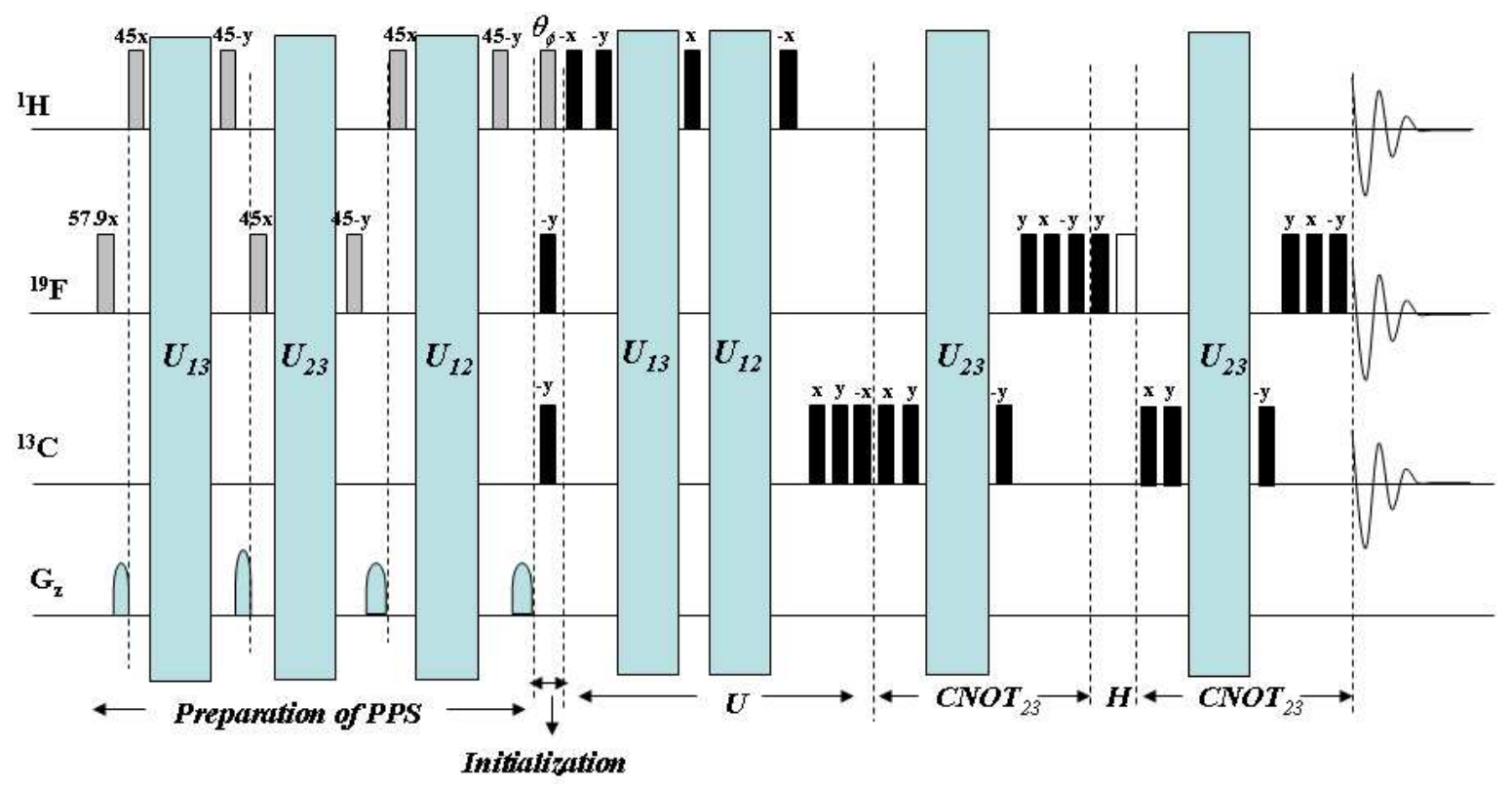

(a)

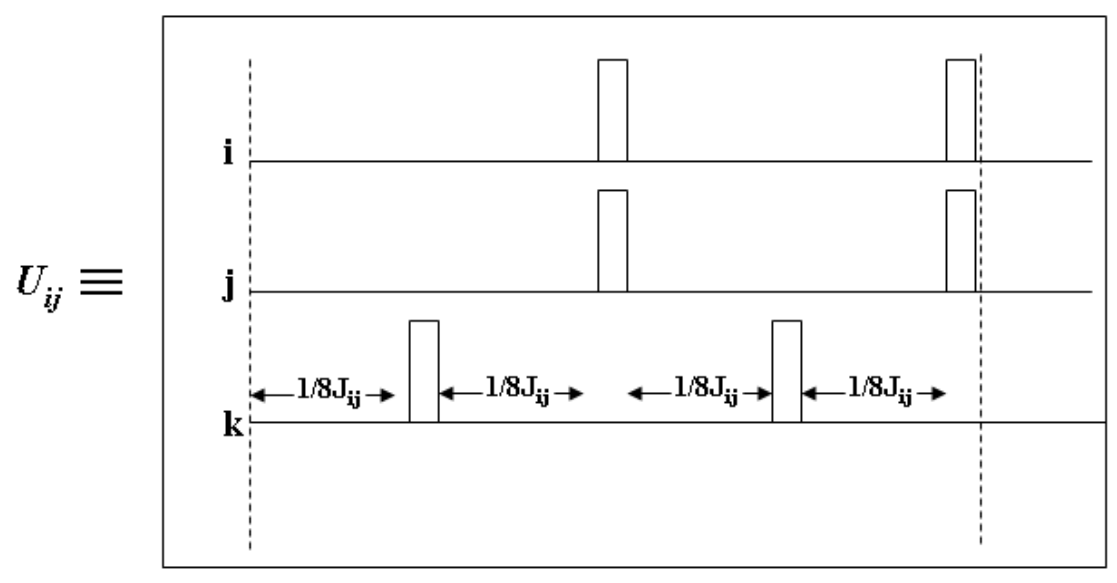

(b)

FIG. 3: (a) NMR pulse sequence for the implementation of the no-hiding theorem using the state randomization. The filled and the empty boxes respectively represent $\pi / 2$ and $\pi$ pulses while the grey boxes represent pulses with flip angles on the top. The phase of a pulse is given on top of the pulse for $\pi / 2$ and $\pi$ pulses and are subscripts of angles for other flip angle pulses. Gz is the z-gradient pulse used to destroy all unwanted transverse magnetization. For each $(\theta, \phi)$ pair three identical experiments are performed to observe each qubit independently. (b) The pulse sequence corresponding to the $U_{i, j}$ operator. 
and (v) finally the measurement (reconstruction of density matrices via tomography). These sequence of steps can be represented by schematic use of several $U_{i j}$ blocks (shown in light grey). During the application of $U_{i j}$ the system evolves under the scalar coupling $J_{i j}$ between the spins $i$ and $j$ for a time period of $1 / 2 J_{i j}$. They create two spin order modes from a single spin transverse mode and vice versa. The $U_{i j}$ is shown in an expanded manner in Fig. 3(b). The $\pi$ pulses in the center of $U_{i j}$ (Fig. 3(b)) are used to refocus all the chemical shifts and all the scalar couplings except between the spins $i$ and $j$.

The experiment consists of implementing the above five steps on the three qubit system. The initial part of Fig. 3(a) contains preparation of $|000\rangle$ pseudo pure state (PPS) by spatial averaging method [22]. The initial state $|\psi\rangle_{1}|A\rangle_{23}$ (see (4)) is prepared from $|000\rangle$ PPS by applying a $(\theta)_{\phi}$ pulse on the first spin and $[\pi / 2]_{-y}$ pulses (Hadamard gates) on the second and the third spins, respectively. The $(\theta)_{\phi}$ pulse in NMR is represented by the operator

$$
U_{\theta, \phi}=\left(\begin{array}{cc}
\cos (\theta / 2) & -i e^{-i \phi} \sin (\theta / 2) \\
-i e^{i \phi} \sin (\theta / 2) & \cos (\theta / 2)
\end{array}\right)
$$

which transforms the state $|0\rangle$ to the superposition state $|\psi\rangle$, i.e.,

$$
|0\rangle \rightarrow \cos (\theta / 2)|0\rangle+e^{i(\phi-\pi / 2)} \sin (\theta / 2)|1\rangle=\alpha|0\rangle+\beta|1\rangle=|\psi\rangle .
$$

The Hadamard gate $[\pi / 2]_{-y}$ on the second and third qubits transforms $|00\rangle$ to $|A\rangle$. Then, we need to perform the randomization operation $U$ which is given by

$$
U=\left(\begin{array}{cccccccc}
1 & 0 & 0 & 0 & 0 & 0 & 0 & 0 \\
0 & 0 & 0 & 0 & 0 & 1 & 0 & 0 \\
0 & 0 & 0 & 0 & 0 & 0 & 1 & 0 \\
0 & 0 & 0 & 1 & 0 & 0 & 0 & 0 \\
0 & 0 & 0 & 0 & 1 & 0 & 0 & 0 \\
0 & 1 & 0 & 0 & 0 & 0 & 0 & 0 \\
0 & 0 & -1 & 0 & 0 & 0 & 0 & 0 \\
0 & 0 & 0 & 0 & 0 & 0 & 0 & -1
\end{array}\right)
$$

Conversion of this matrix into NMR pulse sequence has been achieved here by the use of a novel algorithmic technique for efficient decomposition of the unitary operators in NMR developed in our laboratory by Ajoy et al [23]. This method uses graphs of a complete set of base operators and develops an algorithmic technique for finding the decomposition of a given unitary operator into basis operators and their equivalent pulse sequences. The equivalent pulse sequence for the unitary operator given in $\mathrm{Eq}(10)$ can be expressed as

$$
U=\exp \left(-i \frac{\pi}{4} \mathbf{1}\right) \exp \left(i \frac{\pi}{2} I_{3 z}\right) \exp \left(-i \pi I_{1 y} I_{2 z}\right) \exp \left(-i \pi I_{1 z} I_{3 z}\right) \exp \left(i \frac{\pi}{2} I_{1 x}\right) \exp \left(i \frac{\pi}{2} I_{1 z}\right) .
$$

The first term yields only an overall phase and can be neglected. The second and last terms are $\pi / 2$ rotations of the third and the first qubits, respectively about the z-axis. The z-rotation is achieved by the composite pulse $[\pi / 2]_{-z}^{i}=[\pi / 2]_{x}^{i}[\pi / 2]_{-y}^{i}[\pi / 2]_{-x}^{i}$, where $i(i=1,2,3)$ refers to various qubits. The fourth term is $U_{13}$ and the third term can be converted to $U_{12}$ by converting $I_{1 y}$ to $I_{1 z}$ using the pulse $[\pi / 2]_{-x}[\pi / 2]_{y}[\pi / 2]_{x}$. The final pulse sequence is obtained as

$$
[\pi / 2]_{-z}^{3}[\pi / 2]_{-x}^{1} U_{12}[\pi / 2]_{x}^{1} U_{13}[\pi / 2]_{-x}^{1}[\pi / 2]_{-z}^{1}
$$


Here, pulses are always applied from right to left.

The next step is extraction of quantum information $|\psi\rangle$ from the ancilla qubits. As we have seen this can be achieved by applying two CNOT gates and one Hadamard gate (Fig. 1 and 3). $\mathrm{CNOT}_{23}$ gate in NMR is realized by the following pulse sequence [24]

$$
[\pi / 2]_{-y}^{2}[\pi / 2]_{x}^{2}[\pi / 2]_{y}^{2}[\pi / 2]_{-y}^{3} U_{23}[\pi / 2]_{y}^{3}[\pi / 2]_{x}^{3} .
$$

Finally, we need to do measurements to confirm our result. All the qubits are directly observed at the end of the computation and no measuring pulse is required. The missing information about $|\psi\rangle$ is actually found in the state of the third qubit. This requires taking the trace over the first and the second qubits. One of the ways of taking this trace is to decouple the first and the second qubits while observing the third qubit [25]. However, this leads to excessive sample heating [25]. We have therefore performed the trace numerically by measuring all the three qubits and appropriately adding the signal intensities [26].

The full density matrix of the output state $\left|\Psi_{\text {out }}\right\rangle$ (with the states ordered as 000, 001, $010,011,100,101,110,111)$ is given by

$$
\left|\Psi_{\text {out }}\right\rangle\left\langle\Psi_{\text {out }}\right|=\left(\begin{array}{cccccccc}
\alpha^{2} & \alpha \beta^{*} & 0 & 0 & 0 & 0 & \alpha^{2} & \alpha \beta^{*} \\
\beta \alpha^{*} & \beta^{2} & 0 & 0 & 0 & 0 & \beta \alpha^{*} & \beta^{2} \\
0 & 0 & 0 & 0 & 0 & 0 & 0 & 0 \\
0 & 0 & 0 & 0 & 0 & 0 & 0 & 0 \\
0 & 0 & 0 & 0 & 0 & 0 & 0 & 0 \\
0 & 0 & 0 & 0 & 0 & 0 & 0 & 0 \\
\alpha^{2} & \alpha \beta^{*} & 0 & 0 & 0 & 0 & \alpha^{2} & \alpha \beta^{*} \\
\beta \alpha^{*} & \beta^{2} & 0 & 0 & 0 & 0 & \beta \alpha^{*} & \beta^{2}
\end{array}\right) .
$$

Eq (14) contains two single quantum terms of amplitude $\alpha \beta^{*}$ on the third qubit $\left(\sigma_{12}, \sigma_{78}\right.$, and complex conjugate (cc)) which are directly observable. Since both the single quantum coherence of the third spin are represented by $\alpha \beta^{*}$, they are in phase with each other. We note that no single quantum (SQ) coherence of spins 1 and 2 are present as they are in the Bell state. There are two double quantum (DQ) terms of amplitude $\alpha^{2} \& \beta^{2}\left(\sigma_{17} \& \sigma_{28}\right.$ and cc) and one triple quantum (TQ) term of amplitude $\alpha \beta^{*}\left(\sigma_{18}\right.$ and cc) which are not directly observable. They have been observed ( for tomography) by converting them to observable single quantum term [27].

The results are summarized in Figs (2) and (4). We have measured a total of 325 input states arranged on a 13 by 25 rectangular grid of $\theta(0 \rightarrow \pi)$ and $\phi(0 \rightarrow 2 \pi)$ values with a spacing of $15^{0}$. The experimental spectra of the input and output states for $\theta=\pi / 2, \phi=0$ are respectively given in Fig. 2(b) and Fig. 2(c). Similarly, for $\theta=\phi=\pi / 2$ these are respectively given in Fig. 2(d) and Fig. 2(e). The receiver phase is set such that we get positive absorption lines for the input state $\theta=\phi=\pi / 2$. For each input state we have separately measured the total NMR signal (integration of the entire multiplet) observed from all the spins 1, 2 and 3 and their real components are plotted in Fig. 4. Fig. 4(a) shows the input state $|\psi\rangle_{1}|00\rangle_{23}$ and Fig. 4(b) shows the output state $\left|\phi^{+}\right\rangle_{12}|\psi\rangle_{3}$. The experimental results (Fig. 4) clearly show sine and cosine modulation of the expected lines, thus showing the coherent quantum oscillations of the original qubit. This ensures that the information about $\alpha$ and $\beta$ has been actually transferred from the first qubit to the third qubit in accordance with the no-hiding theorem.

Additionally we have reconstructed the density matrices of the output state for several values of $\theta$ and $\phi$ by quantum state tomography [27]. The density matrix of the output 

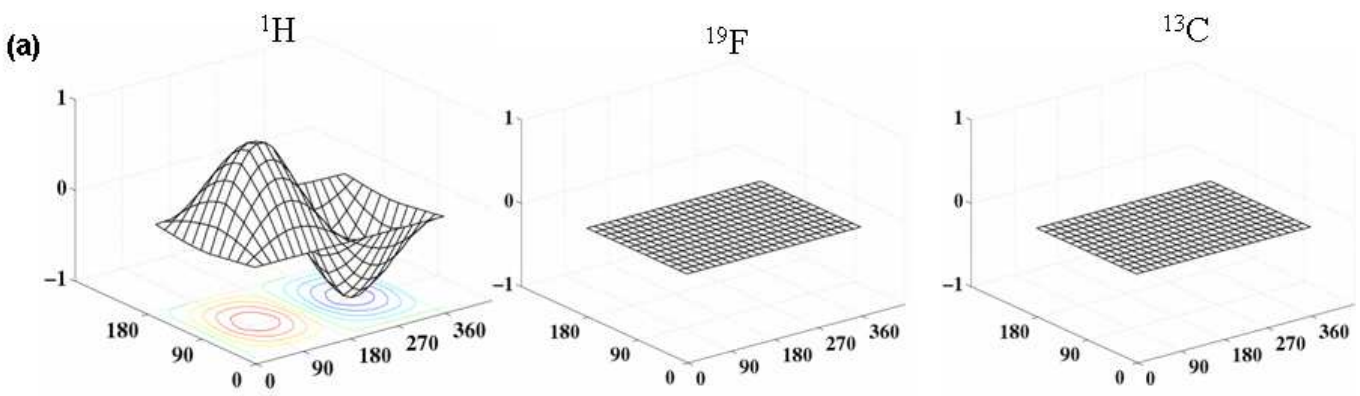

(b)
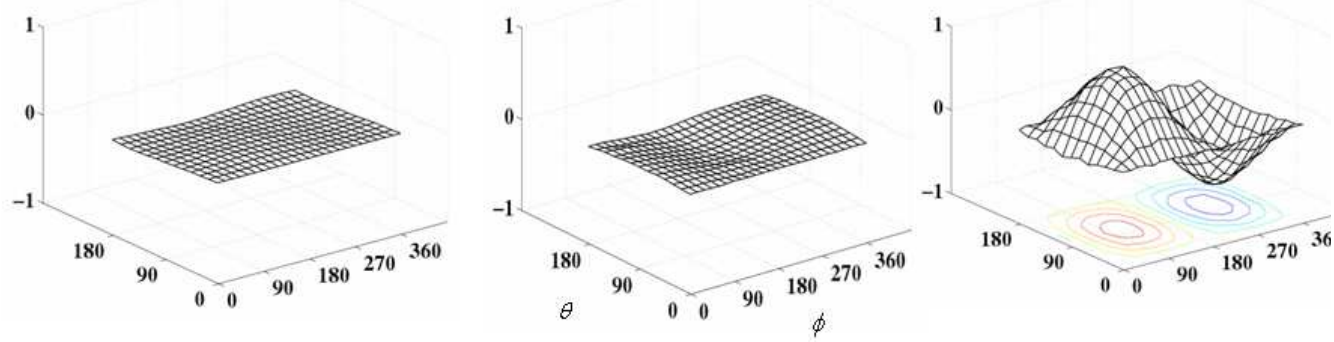

FIG. 4: Summarized experimental results for the no-hiding theorem using the state randomization. The integrals of the real part of the NMR signal from spins ${ }^{1} H,{ }^{19} \mathrm{~F}$ and ${ }^{13} \mathrm{C}$ are shown as mesh and contour plots as a function of $\theta$ and $\phi$. The plots show the expected sine and cosine behavior. (a) Input state $|\psi\rangle_{1}|00\rangle_{23}$. The information about $\theta$ and $\phi$ is encoded in the first spin, and second and third spins are in $|00\rangle$ state. (b) Final output state of three spins $\left.1 / \sqrt{2}\left(|00\rangle_{12}+|11\rangle_{12}\right)|\psi\rangle_{3}\right]$. The information about the state $|\psi\rangle$ has been transferred from the first qubit to the third qubit. The first and second qubits are in the Bell state.

state for $\theta=\phi=\pi / 2$ is shown in Fig. 5. The experimental state tomography confirms the theoretical output state as given in (14). The fidelity of the measurement has been evaluated for several values of $\theta \& \phi$ using the parameters "average absolute deviation $\langle\Delta X\rangle$ " and the "maximum absolute deviation $\Delta X_{\max }$ " as defined by

$$
\begin{gathered}
\langle\Delta X\rangle=\frac{1}{N^{2}} \sum_{i, j=1}^{N}\left|x_{i, j}^{T}-x_{i, j}^{E}\right|, \\
\text { and } \Delta X_{\max }=\operatorname{Max}\left|x_{i, j}^{T}-x_{i, j}^{E}\right|, \forall i, j \in\{1, N\},
\end{gathered}
$$

where $x_{i, j}^{T}, x_{i, j}^{E}$ are the theoretical and the experimental elements [21]. The average absolute deviation ( for three $\theta \& \phi$ values) $\langle\Delta X\rangle$ was found to be $\sim 2 \%$ and the maximum absolute deviation $\Delta X_{\max }$ was found to be $\sim 5 \%$.

In our experiment the reduced density matrices of the first two qubits of the output states $\left(\left|\phi^{+}\right\rangle\right.$of Fig. 1) have also been tomographed to observe the fidelity of the Bell states in the first two qubits at the end of the measurement. Fig. 6(a) contains the Bell state $\left|\phi^{+}\right\rangle_{12}$ for the input state with parameters $\theta=\phi=\pi / 2$ and Fig. 6(b) contains $\left|\phi^{+}\right\rangle_{12}$ for the input 


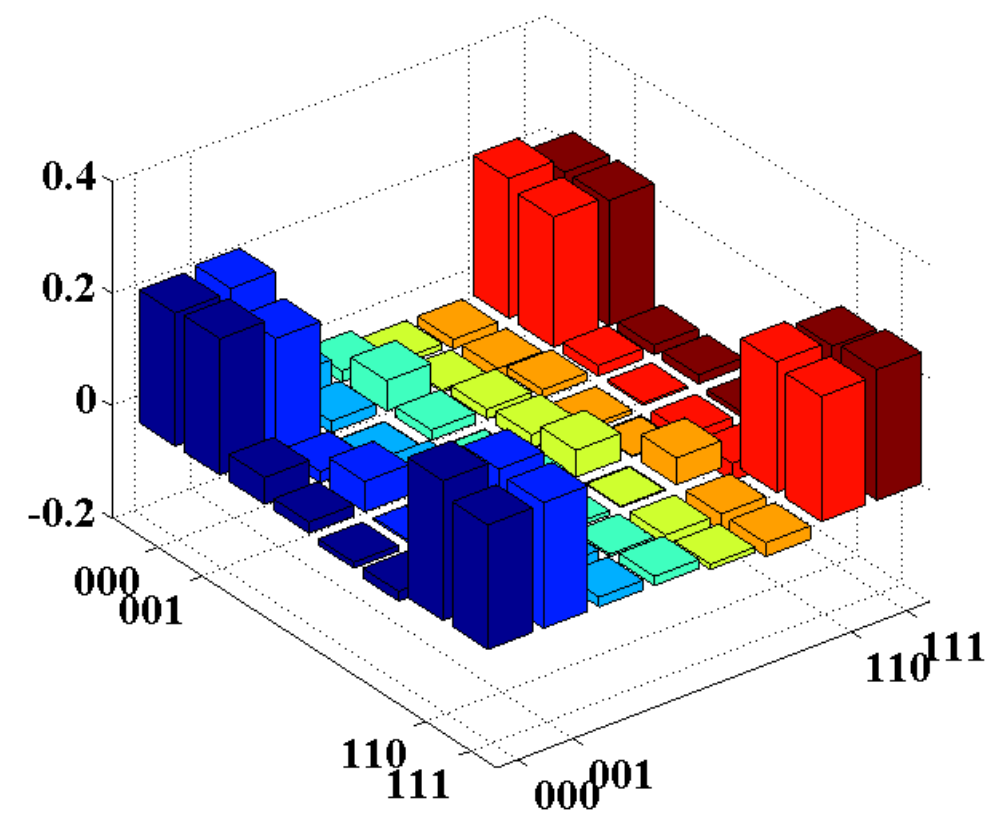

FIG. 5: Tomographed density matrix of the output state for $\theta=\phi=\pi / 2$.

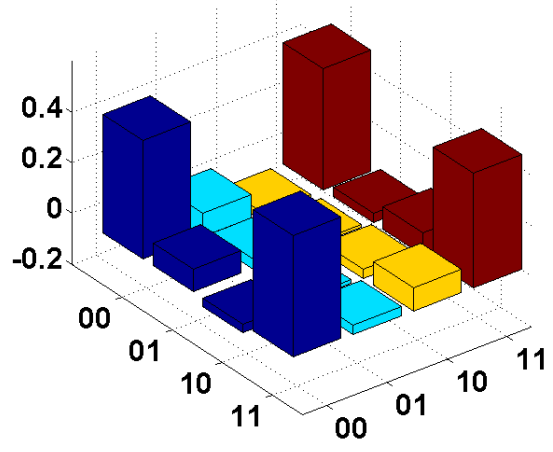

(a)

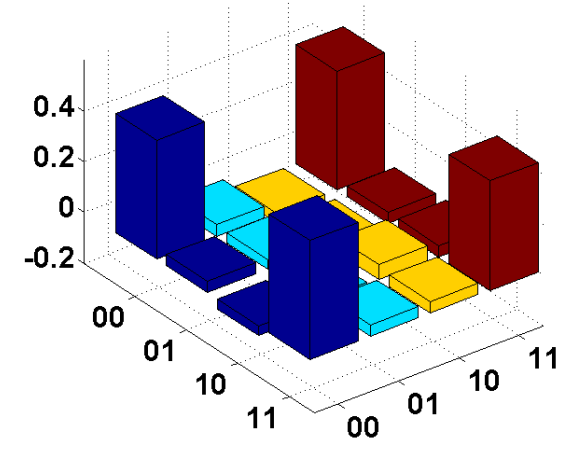

(b)

FIG. 6: The output density matrix for the first two qubits showing that they are in the Bell state $\left|\phi^{+}\right\rangle$. (a) For $\theta=\phi=\pi / 2$ (b) For $\theta=\pi / 2$ and $\phi=\pi$

state with parameters $\theta=\pi / 2, \phi=0$. Fig. 6 confirms that the first two qubits remain in the Bell state irrespective of the changes in $(\theta, \phi)$ and the original information about $(\theta, \phi)$ have been transferred to the third qubit. The average absolute deviation $\langle\Delta X\rangle$ was found to be $\sim 5 \%$ and the maximum absolute deviation $\Delta X_{\max }$ was found to be $\sim 7 \%$. The experimental errors can originate from rf inhomogeneities, imperfect calibration of rf pulses 
and decoherence. However, in the present experiment the decoherence errors are likely to be small, since the total experimental time (Fig. 3) is $\sim 30 \mathrm{msec}$ while the shortest $T_{2}$ (of ${ }^{19} \mathrm{~F}$ ) of the sample is 700 msec.

To conclude, we have performed a proof-of-principle demonstration of the no-hiding theorem and addressed the question of missing information on a 3-qubit NMR quantum information processor. Using the state randomization as a prime example of the bleaching process we have found that the original quantum information which is missing from the first qubit, indeed can be fully recovered from the ancilla qubits. We have encoded the quantum information in ${ }^{1} \mathrm{H}$ spin and later it has been fully reconstructed experimentally from ${ }^{13} \mathrm{C}$ spin using the NMR pulse sequence. No information is found to be hidden in the bipartite correlations between the original qubit and the ancilla qubits. To the best of our knowledge this is the first experimental verification of this fundamental theorem of quantum mechanics.

Randomization of quantum state is an important primitive in private quantum channels which permits private transmission of quantum information using a shared classical key. One can randomize $n$-qubits perfectly using $2 n$ classical bits. This is often stated as a quantum analog of the classical one-time pad. However, the no-hiding theorem reveals an important difference between the classical one-time pad and the quantum analog. In the classical onetime pad when information is encrypted using a random key, then the original information is neither in the message nor in the key but hidden in the correlations. In the quantum case, when the original information is missing it simply resides in the key (in the unitary version in the two qubit ancilla) from which one can recover the original qubit. In this sense our experiment also demonstrates an important difference between the classical one-time pad and its quantum analog. The no-hiding theorem not only holds in the perfect case (where information completely disappears) but also it is robust to imperfections. We hope that NMR will continue to be an excellent test bed for verification of quantum mechanical predictions and our work will inspire others to test the no-hiding theorem in the context of quantum teleportation and thermalization.

Acknowledgements: We thank P. Rungta for useful discussions.

[1] Wootters, W. K., \& Zurek, W. H. A single quantum can not be cloned. Nature 299, 802 (1982).

[2] Pati, A. K., \& Braunstein, S. L. Impossibility of deleting an unknown quantum state. Nature 404, 164 (2000).

[3] Jozsa, R., Illustrating the concepts of quantum information. IBM J. Res. Dev. 48, 79 (2004).

[4] Braunstein, S. L., \& Pati, A. K. Quantum information cannot be completely hidden in correlations: Implications for the Black hole information paradox. Phys. Rev. Lett. 98, 080502 (2007).

[5] Braunstein, S. L. Quantum teleportation without irreversible detection. Phys. Rev. A 53, 1900 (1996).

[6] Braunstein, S. L., Lo, H. K., \& Spiller, T. Forgetting qubits is hot to do. Unpublished manuscript, (1999). 
[7] Ambainis, A., Mosca, M., Tapp, A., \& de Wolf, R. Private quantum channels. IEEE Symposium on Foundations of Computer Science (FOCS), 547-553 (2000).

[8] Popescu, S., Short, A. J., \& Winter, A. Entanglement and the foundations of statistical mechanics. Nature Phys. 2, 754 (2006).

[9] Hawking, S. W. Black hole explosion. Nature 248, 30 (1974).

[10] Zurek, W. H. Decoherence and the Transition from Quantum to Classical. Physics Today 44, 36-44 (1991).

[11] Vernam, G. S., J. American Inst. Elec. Eng. 55, 109 (1926).

[12] Shannon, C. E., Bell Systems Tech. J. 28, 656 (1949).

[13] Landauer, R., IBM J. Res. Dev. 3, 183 (1961).

[14] Chuang, I. L., Gershenfeld, N., and Kubinec, M. Experimental implemnattaion of fast quantum searching. Phys. Rev. Lett. 80, 3408 (1998).

[15] Vandersypen, L. M. K., Steffen, M., Breyta, G., Yannoni, C. S., Sherwood, M. H., and Chuang, I. L. Experimental realization of Shor's quantum factoring algorithm using nuclear magnetic resonance. Nature 414 883-887 (2001).

[16] Nielsen, M. A, Knill, E., Laflamme, L. Complete quantum teleportation using nuclear magnetic resonance. Nature 396, 52-55 (1998).

[17] Mitra, A., Ghosh, A., Das, R., Patel, A., \& Kumar, A. Experimental implementation of local adiabatic evolution algorithms by an NMR quantum information processor. J. Mag. Resonance 177, 285-298 (2005).

[18] Peng, X., Liao, Z., Xu, N., Qin, G., Zhou, X., Suter, D., \& Du, J. Quantum Adiabatic Algorithm for Factorization and Its Experimental Implementation. Phys. Rev. Lett. 101, 220405 (2008).

[19] Du, J., Xu, N., Peng, X., Wang, P., Wu, S., and Lu, D. NMR implementation of a molecular Hydrogen quantum simulation with adiabatic state preparation. Phys. Rev. Lett. 104, 030502 (2010).

[20] Samal, J. R., Gupta, M., Panigrahi, P., and Kumar, A. Non-destructive discrimination of Bell states by NMR using a single ancilla qubit. J. Phys. B: At. Mol. Opt. Phys. 43, 095508 (2010).

[21] Mitra, A., Supriya, K., and Kumar, A. Experimental implementation of a 3-qubit quantum game using NMR quantum information processor, J. Mag. Resonance 187, 306-313 (2007).

[22] Cory, D. G., Fahmy, A. F., \& Havel, T. F. Ensemble Quantum computing by NMR spectroscopy. Proc. Natl. Acad. Sci. 94, 1634 (1997).

[23] Ajoy, A., Samal, J. R., Rungta, P., Kumar, A. Algorithmic technique for efficiently decomposing unitary operatins in NMR quantum computation. Manuscript under preaparation (2010).

[24] Childs, A. M., Chuang, I. L., \& Leung, D. W. Realization of quantum process tomography in NMR, Phys. Rev. A. 64, 012314 (2001).

[25] Sharf, Y., Cory, D. G., Somaroo, S. S., Knill, E., Laflamme, R., Zurek, W. H., \& Havel, T. F. A study of quantum error correction by geometric algebra and liquid-state NMR spectroscopy, Mol. Phys. 98, 1347 (2000).

[26] Cummins, H. K., Jones, C., Furze, A., Soffe, N. F., Mosca, M., Peach, J. M., \& Jones, J. A. Approximate quantum clonig with nuclear magnetic resonance, Phys. Rev. Lett. 88187901 (2002).

[27] Mitra, A., Quantum algorithms using nuclear magnetic resonance quantum information processor, PhD Thesis, Indian Institute of science, Bangalore (2007). 